\title{
(6) OPEN ACCESS \\ Evaluation of clinically significant adverse events in patients discharged from a tertiary-care emergency department in Taiwan
}

\author{
Lee-Min Wang, ${ }^{1,2,3}$ Chorng-Kuang How, ${ }^{2,4}$ Ming-Chin Yang, ${ }^{3}$ Syi Su, ${ }^{3}$ \\ Chii-Hwa Chern ${ }^{2,4}$
}

${ }^{1}$ Department of Emergency Medicine, Taichung Veterans General Hospital, Taiwan, ROC ${ }^{2}$ Emergency Medicine, School of Medicine, National Yang-Ming University, Taiwan, ROC ${ }^{3}$ Institute of Health Care Organization Administration, National Taiwan University, Taiwan, ROC

${ }^{4}$ Department of Emergency Medicine, Taipei Veterans General Hospital, Taiwan, ROC

\section{Correspondence to}

Dr Chii-Hwa Chern, Department of Emergency Medicine, Taipei Veterans General Hospital, Taiwan 112, ROC; chchern2002@yahoo.com.tw

Accepted 21 February 2012

Published Online First

20 March 2012

\begin{abstract}
Objective To investigate the reasons for the occurrence of clinically significant adverse events (CSAEs) in emergency department-discharged patients through emergency physicians' (EPs) subjective reasoning and senior EPs' objective evaluation.
\end{abstract}

Design This was a combined prospective follow-up and retrospective review of cases of consecutive adult nontraumatic patients who presented to a tertiary-care emergency department in Taiwan between 1 September 2005 and 31 July 2006. Data were extracted from 'onduty EPs' subjective reasoning for discharging patients with CSAEs (study group) and without CSAEs (control group)' and 'objective evaluation of CSAEs by senior EPs, using clinical evidences such as recording history, physical examinations, laboratory/radiological examinations and observation of inadequacies in the basic management process (such as recording history, physical examinations, laboratory/radiological examinations and observation) as the guide'. Subjective reasons for discharging patients' improvement of symptoms, and the certainty of safety of the discharge were compared in the two groups using $\chi^{2}$ statistics or $t$ test.

Results Of the 20512 discharged cases, there were 1370 return visits $(6.7 \%, 95 \% \mathrm{Cl} 6.3 \%$ to $7 \%)$ and 165 CSAEs due to physicians' factors $(0.82 \%, 95 \% \mathrm{Cl} 0.75 \%$ to $0.95 \%)$. In comparisons between the study group and the control group, only some components of discharge reasoning showed a significant difference $(p<0.001)$. Inadequacies in the basic management process were the main cause of CSAEs (164/165).

Conclusion The authors recommended that EP follow-up of the basic management processes (including history record, physical examination, laboratory and radiological examinations, clinical symptoms/signs and treatment) using clinical evidence as a guideline should be made mandatory.

\section{INTRODUCTION}

Patient safety and medical errors are currently major controversial issues. Due to various extrinsic factors, the emergency department (ED) is one of the most common sites within the hospital for development of adverse events. ${ }^{1-3}$ Several studies have shown that there is a risk of occurrence of an 'adverse event', defined as an injury due to treatment, ranging between $2.5 \%$ and $11 \%$ of all hospitalisations. ${ }^{2-12}$ Approximately one half of all adverse events are potentially preventable. ${ }^{45}$ The ED has been identified as a hot spot where adverse events are more likely to be attributable to error. ${ }^{5}$ However, few studies have measured the risk of adverse events occurring after the discharge of hospitalised medical patients. ${ }^{1-5}$ In fact, little is known about the ED adverse events/errors.

Croskerry has stated that cognitive errors underlie most diagnostic errors that are made in the course of clinical decision making in the ED. ${ }^{3}$ Knowledge-based cognitive behaviour involves interpreting and understanding novel situations and problems against a background of specific domain knowledge (eg, integrating the presenting complaint, medical history, physical examination and laboratory findings). Therefore, it would seem to be more useful to recommend that on-duty emergency physicians (EPs) undertake more detailed documentation of patient history, physical examination and medical decision making to prevent adverse events than to try to change the thresholds of the basic management process (BMP). Therefore, in this study we investigated the EPs' reasons for discharge of patients with clinically significant adverse events (CSAEs). CSAEs included major operative conditions, major medical conditions, significant deterioration of clinical conditions and prolonged hospital stay (>3 days). ${ }^{4} 9$ We based our observations on EPs' subjective reasoning for discharging patients and senior EPs' objective evaluations.

\section{MATERIALS AND METHODS}

The study was conducted at a university-affiliated teaching hospital with an annual ED census of over 82000 in Taiwan, and the protocol was approved by the institutional review board of the study hospital. All participating physicians signed a consent form agreeing to be interviewed before being enrolled. From 1 September 2005 to 31 July 2006 , we conducted a combined prospective followup and retrospective review study. This study was divided into two parts: a prospective investigation based on 'on-duty EPs' subjective reasoning for discharging patients with CSAEs and without CSAEs', and a retrospective analysis using 'objective evaluation of CSAEs by senior EPs' (figure 1). The main outcome measures were the EPs' subjective reasons for discharging patients with CSAEs, and an objective analysis of inadequacies in the BMP model such as those involving history record, physical examination, laboratory and radiological examinations, clinical symptoms/signs and treatment of patients with CSAEs. We collected cases of return visits within 3 days daily at regular intervals (8:00, 20:00 and 24:00), using the hospital's patient computer database, which is updated in real-time. 
Figure 1 (A) Prospective follow-up and $(B)$ retrospective review of cases of return visit within 3 days. CSAE, clinically significant adverse event; ED, emergency department; EP, emergency physician.

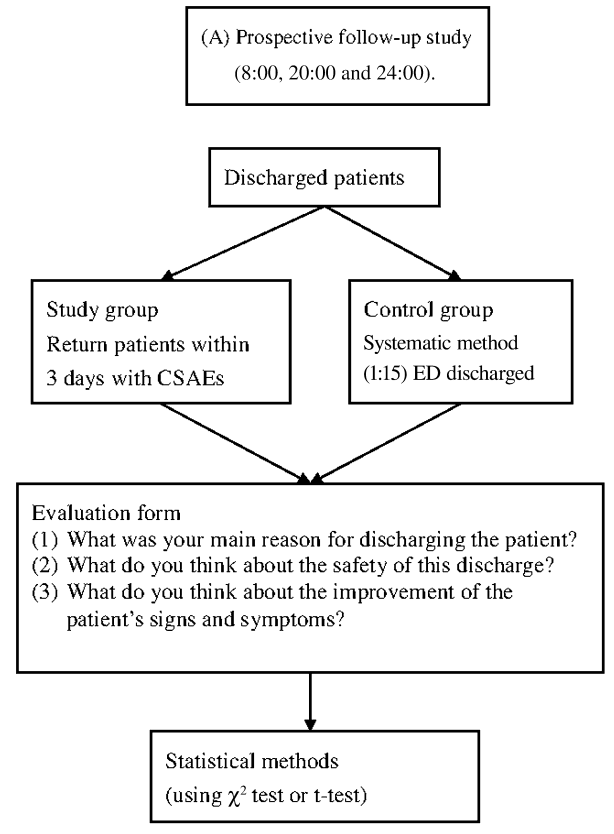

\section{EPs' subjective reasons for discharging patients with CSAEs and without CSAEs}

We collected information on cases of CSAEs (study group) from return visits. At the same time, we also collected data on consecutive ED patients who were discharged without CSAEs (control group) using a systematic method (1:15 ED-discharged patients). Trauma and paediatric cases were excluded. We used the open-question method with the aid of an interview form, as shown in figure 2 and figure $1 \mathrm{~A}$. The main purpose of the interview form was to allow EPs to express their subjective reasons for discharging ED patients and to subjectively evaluate the safety of their patients and any improvements in the patients' symptoms/signs. ${ }^{6}$

\section{Objective evaluation of CSAEs by senior EPs}

Because of the possibility of inadequate documentation in the first visit chart, we checked and confirmed the opinion of EPs who saw patients in their second visits. After the interview and collection of any relevant data by two assistants, two EPs independently evaluated the return visit cases to determine if the cases had CSAEs and the causes of the CSAEs. In cases of disagreement, the two EPs met to reach a consensus or consulted with a third EP. We excluded the following conditions that were not considered a physician's factor (responsibility). 'Disease process' meant a condition due to a disease factor itself, not due to a physician's error. 'A different disease' meant a different disease was diagnosed in the second visit that was not clinically associated with that of the first visit. 'No need of hospitalisation' was defined as a clinical condition that did not require hospitalisation or was not expected to have any major adverse effect if patients were not hospitalised. 'The need of regular treatment' was the clinical condition that required regular procedures. After the previously mentioned conditions were excluded, the causes of CSAEs would be considered as 'physicians' factors' if we could find clinical evidence of inadequacies in the BMP or definite physicians' errors, and the causes would be considered part of the disease process if we could not (the analytical process is described below). When EPs made definite errors but no inadequacies in the BMPs were identified, then the cause of CSAEs was defined as 'judgement defect'.
Every facet of basic management involves a judgement. In this study, we emphasised the importance of the BMP, which includes history taking, physical examinations, appropriate tests and x-ray examinations, and re-evaluation and observation, and considered that completing this process is a basic requirement of EPs. Therefore, absence of any one component in the process is not thought to be a 'pure' judgement error. The objective review process to determine CSAEs and also their causes, as shown in figure $1 \mathrm{~B}$, uses a systematic method to ascertain whether there was clinical evidence to indicate insufficiencies in the BMP ${ }^{13} 14$ or a definite error. Any insufficiency in this process was considered to be an error of 'not done' (a neglect of the basic requirement) and not a 'judgement error'.

\section{Statistical methods}

Subjective reasons for discharging patients, including improvement of symptoms and the certainty of safety of the discharge, were compared in the two groups using $\chi^{2}$ statistics or t-test. We used Microsoft Excel for data filing and AcaStat software for analysis (AcaStat Software, Ashburn, Virginia, USA).

\section{RESULTS}

There were 20512 cases of patients who were directly discharged from the ED, with 1370 (6.8\%, 95\% CI 6.3\% to 7\%) return visits and 165 CSAEs $(0.82 \%, 95 \%$ CI $0.75 \%$ to $0.95 \%)$.

\section{Collection of data for patients with CSAEs}

Of the 1370 cases of return visits, excluding cases with mild clinical conditions and hospitalisations for $\leq 3$ days, 420 cases met one of the set of criteria for CSAEs, and most cases included hospitalisations for $>3$ days. The causes of these possible CSAEs were physicians' factors (165), disease progress (69), diseases needing definite treatment (65), patients' factors (51), factors related to the disease itself (38), different diseases (25) and unexpected treatment complications (7). The definitions of these conditions are given in the methods section. The actual CSAEs were defined as CSAEs due to physicians' factors, implying errors were present. After excluding cases due to 'different diseases', 'diseases needing definite treatment', 


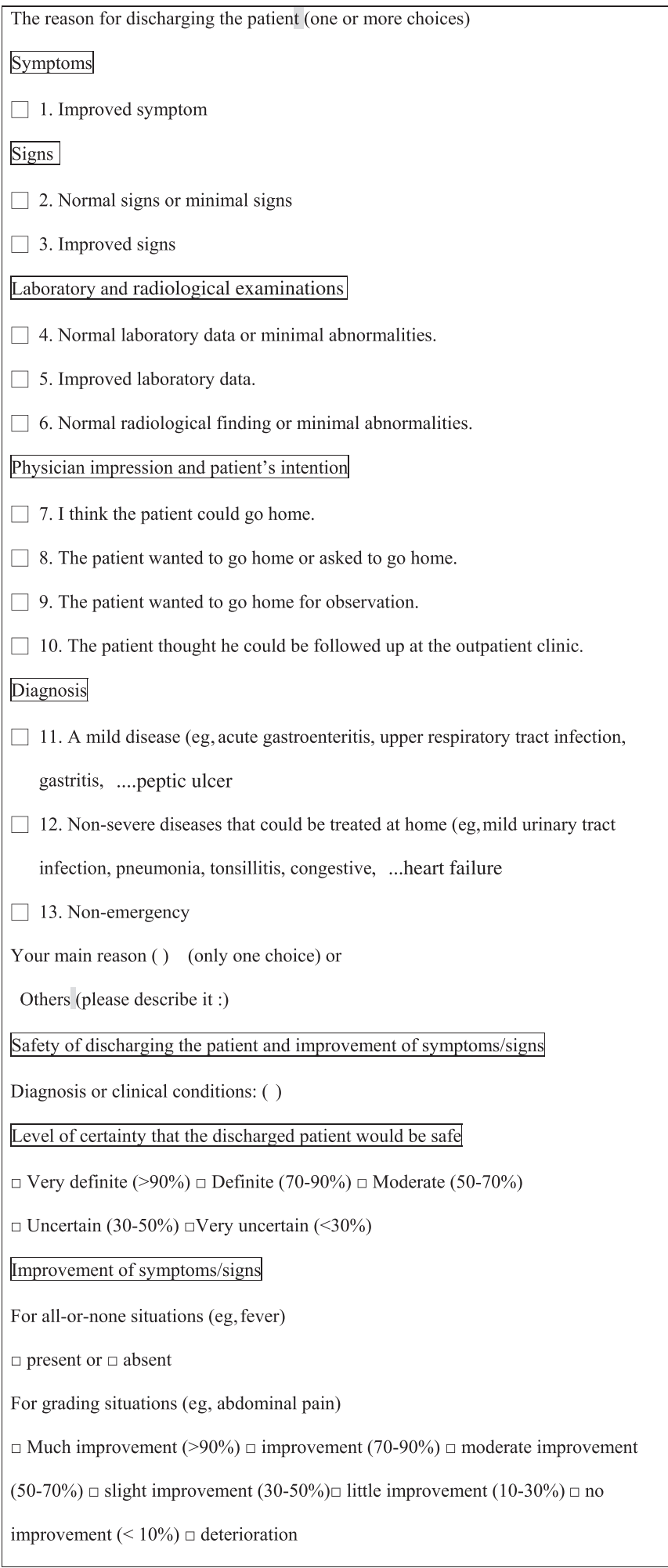

Figure 2 The evaluation form for discharged patients.

'patients' factors', 'factors related to the disease itself', 'unexpected treatment complications', and 'disease factors' (186 cases in total), an objective review showed physicians' factors (real CSAEs by definition) accounted for 165 cases.

Comparisons between the control group (regularly discharged patients) and the study group (patients with CSAEs)

Some components of discharge reasoning including the main reasons for discharging patients' and 'the certainty level of safety of discharging patients' reached statistical significance $(\mathrm{p}<0.001)$ when the CSAEs group was compared with the control group, as shown in tables 1 and 2. As regards the main reason for discharge, the majority of EPs thought their patients had mild disease status and their symptoms had improved. With regard to discharge based on patients' wish and physicians' impression (EPs' impression or consultants' opinion) as the main reasons, significant differences in both groups were noted $(p<0.001)$. 'Patients' wish to be discharged' was not an uncommon reason and might suggest EPs just followed the wishes of the patients even if the patient's condition was still uncertain. Some EPs discharged patients on the advice of consultants, which might have greatly influenced the EPs' independent judgement.

Regarding the certainty level of safety of discharge, a majority of EPs thought their patients had high safety in discharge

There were significant differences in $50-70 \%$ of safety certainty issues between non-CSAEs and CSAEs $(p<0.001)$ (table 2). Using these main parts to discriminate the CSAEs and the control group patients would be impossible in clinical conditions, especially when these main parts were appropriate reasons for discharge. In both groups, few patients were thought to have a low level of safety when discharged.

Regarding subjective improvement of symptoms, the majority of EPs thought their patients had shown much symptomatic improvement or had minimal initial symptoms

There were no significant differences in symptomatic improvement for non-CSAEs or CSAEs (table 3). Clinically, using the main parts to discriminate the CSAEs and the control group patients would be impossible.

Subjective reasons for discharging patients with CSAEs and objective review to ascertain the causes of CSAEs in discharged patients

For most discharged patients with CSAEs, EPs thought they were cases of mild disease and/or improved symptoms. The main reasons for discharge were 'mild disease' and 'improved symptoms' in $68 \%$ of the CSAEs cases (table 4). However, in our

Table 1 The main reasons for discharge of the patient

\begin{tabular}{|c|c|c|c|}
\hline & $\begin{array}{l}\text { Non-CSAEs }(\mathrm{N}=2658) \\
\mathrm{N}(\%)\end{array}$ & $\begin{array}{l}\text { CSAEs (N=165) } \\
N(\%)\end{array}$ & p Value \\
\hline \multicolumn{4}{|c|}{ Improved symptom } \\
\hline No & $1735(65.3)$ & $112(67.9)$ & \multirow[t]{2}{*}{$0.550 \dagger$} \\
\hline Yes & $923(34.7)$ & $53(32.1)$ & \\
\hline \multicolumn{4}{|c|}{ Normal physical examinations } \\
\hline No & $2611(98.2)$ & $161(97.6)$ & \multirow[t]{2}{*}{$0.539 \neq$} \\
\hline Yes & $47(1.8)$ & $4(2.4)$ & \\
\hline \multicolumn{4}{|c|}{ Normal laboratory/radiological examinations } \\
\hline No & $2581(97.1)$ & $158(95.8)$ & \multirow[t]{2}{*}{$0.338 \neq$} \\
\hline Yes & $77(2.9)$ & $7(4.2)$ & \\
\hline \multicolumn{4}{|c|}{ Mild disease } \\
\hline No & $1621(61.0)$ & $106(64.2)$ & \multirow[t]{2}{*}{$0.453 \dagger$} \\
\hline Yes & $1037(39.0)$ & $59(35.8)$ & \\
\hline \multicolumn{4}{|c|}{ Emergency physician/consultant's opinion } \\
\hline No & $2194(82.5)$ & $45(17.3)$ & \multirow[t]{2}{*}{$<0.001 \dagger$} \\
\hline Yes & $464(17.5)$ & $120(72.7)$ & \\
\hline \multicolumn{4}{|c|}{ Patient's desire to be discharged } \\
\hline No & $2548(95.9)$ & $143(86.7)$ & \multirow[t]{2}{*}{$<0.001 \dagger$} \\
\hline Yes & $110(4.1)$ & $22(13.3)$ & \\
\hline
\end{tabular}


Table 2 The certainty level of safety of discharge

\begin{tabular}{|c|c|c|c|}
\hline & $\begin{array}{l}\text { Non-CSAEs }(\mathrm{N}=2657) \\
\mathrm{N}(\%)\end{array}$ & $\begin{array}{l}\text { CSAEs }(\mathrm{N}=165) \\
\mathrm{N}(\%)\end{array}$ & p Value \\
\hline \multicolumn{4}{|c|}{ Safety $>90 \%$} \\
\hline No & $2205(83.0)$ & 141 (85.5) & \multirow[t]{2}{*}{$0.475 \dagger$} \\
\hline Yes & $452(17.0)$ & $24(14.5)$ & \\
\hline \multicolumn{4}{|c|}{ Safety $>70-90 \%$} \\
\hline No & $787(29.6)$ & $52(31.5)$ & \multirow[t]{2}{*}{$0.668 \dagger$} \\
\hline Yes & $1870(70.4)$ & $113(68.5)$ & \\
\hline \multicolumn{4}{|c|}{ Safety $50-70 \%$} \\
\hline No & $2392(90.0)$ & $131(79.4)$ & \multirow[t]{2}{*}{$<0.001 \dagger$} \\
\hline Yes & $265(10.0)$ & $34(20.6)$ & \\
\hline \multicolumn{4}{|c|}{ Safety $30-50 \%$} \\
\hline No & $2604(98.0)$ & $160(97.0)$ & \multirow[t]{2}{*}{$0.386 \neq$} \\
\hline Yes & $53(2.0)$ & $5(3.0)$ & \\
\hline \multicolumn{4}{|c|}{ Safety $<30 \%$} \\
\hline No & $2640(99.4)$ & $165(100.0)$ & \multirow[t]{2}{*}{$0.620 \ddagger$} \\
\hline Yes & $17(0.6)$ & $0(0.0)$ & \\
\hline
\end{tabular}

observation, EPs were seldom mindful of consultants' tendency to put greater emphasis on their field of expertise and as a result, consultants may not have evaluated the patient as a whole or considered health issues outside their field.

In the objective review of return visit cases, we found four major areas of insufficiency: history taking (55), observation of symptoms (47), laboratory/radiological examination and observation of treatment response (32) and all were due to physicians' factors (table 4). The insufficient observation of clinical symptoms $(28 \%, 47 / 165)$ and insufficient observation of treatment response $(19 \%, 32 / 165)$ were the most important causes of CSAEs due to physicians' factors (table 4). Only one case $(1 / 165$, $0.6 \%$ ) was considered to be purely due to a 'judgement defect'.

\section{DISCUSSION}

Our findings showed that a majority of patients with CSAEs were discharged by EPs who subjectively thought their patients

Table 3 Symptomatic improvement of the patient

\begin{tabular}{|c|c|c|c|}
\hline & $\begin{array}{l}\text { Non-CSAEs }(\mathrm{N}=2664) \\
\mathrm{N}(\%)\end{array}$ & $\begin{array}{l}\text { CSAEs }(\mathrm{N}=165) \\
\mathrm{N}(\%)\end{array}$ & p Value \\
\hline \multicolumn{4}{|c|}{ Much improvement (>90\%) } \\
\hline No & $2048(76.9)$ & $134(81.2)$ & \multirow{2}{*}{$0.234 \dagger$} \\
\hline Yes & $616(23.1)$ & $31(18.8)$ & \\
\hline \multicolumn{4}{|c|}{ Improvement (70-90\%) } \\
\hline No & $2344(88.0)$ & $144(87.3)$ & \multirow[t]{2}{*}{$0.880 \dagger$} \\
\hline Yes & $320(12.0)$ & $21(12.7)$ & \\
\hline \multicolumn{4}{|c|}{ Moderate improvement $(50-70 \%)$} \\
\hline No & $1555(58.4)$ & $100(60.6)$ & \multirow[t]{2}{*}{$0.628 \dagger$} \\
\hline Yes & $1109(41.6)$ & $65(39.4)$ & \\
\hline \multicolumn{4}{|c|}{ Slight improvement $(30-50 \%)$} \\
\hline No & $2320(87.1)$ & $142(86.1)$ & \multirow[t]{2}{*}{$0.794 \dagger$} \\
\hline Yes & $344(12.9)$ & $23(13.9)$ & \\
\hline \multicolumn{4}{|c|}{ Little improvement $(10-30 \%)$} \\
\hline No & $2566(96.3)$ & $156(94.5)$ & \multirow[t]{2}{*}{$0.342 \dagger$} \\
\hline Yes & $98(3.7)$ & $9(5.5)$ & \\
\hline \multicolumn{4}{|c|}{ No improvement $(<10 \%)$ or deterioration } \\
\hline No & $2487(93.4)$ & $149(90.3)$ & \multirow[t]{2}{*}{$0.177 \dagger$} \\
\hline Yes & $177(6.6)$ & $16(9.7)$ & \\
\hline
\end{tabular}

had mild disease, improved symptoms and a high certainty level of safety. In discharges that used patients' wishes and physicians' impressions (EPs' impression or consultants' opinion) as the main reasons, there were significant differences in both groups (non-CSAEs and CSAEs). For the physicians' factors, we found an insufficient observation of clinical symptoms/signs $(28.5 \%, 47 / 165)$ and treatment $(19.4 \%, 32 / 165)$ and inadequacy in recording history $(33.3 \%, 55 / 165)$ were the most common causes of CSAEs. Only one case $(1 / 165,0.6 \%)$ was an error purely due to the judgement factor.

Patients discharged from the ED may be prone to adverse events due to the nature of ED care. EPs usually do not know exactly when or how the patients were treated before their ED visit and may lack their critical information. ${ }^{1}$ Furthermore, the ED is often overcrowded ${ }^{1-3}$ and chaotic. ${ }^{11} \mathrm{ED}$ patients often fully rely on diagnostic and therapeutic interventions, but waiting for differential diagnostic or interventions is inherently risky. ${ }^{2}$ Therefore, preventive methods for measuring adverse events in the ED were developed for the evaluation of these risks. $^{2}$ 7-9

In our previous study, we demonstrated that a quality improvement programme with feedback to physicians of telephone follow-up and resident education, significantly decreased clinically adverse events in ED-discharged patients. ${ }^{9}$ The programme included four basic changes: First, residents needed a high level of education. Second, before discharge of the patient, the emergency attending physician had to examine the patient. Third, a documentation audit was required. ${ }^{9}$ Finally, given the lower success rate in contacting patients before their presentation to the ED with a CSAE, we concluded that a shorter interval of follow-up may be required for certain patients. ${ }^{9}$ In general, ED residents were involved in the majority of the claims. Therefore, resident supervision is definitely needed and is a part of a specific training programme for EPs. ${ }^{10}$ Numerous studies have described the incidence, patterns, and causes of adverse events in patients discharged from the ED using a systematic review process. ${ }^{6-12}$

Our study was compatible with previous studies. First, our findings supported the concept demonstrated in our previous study and in other reports that a complete BMP is important for preventing CSAEs. ${ }^{912} 15$ Inadequacies in the BMP produced 'pseudo-normal results' that interfered with the selection of high-risk patients for a telephone follow-up. ${ }^{9}$ We found that the most important factors leading to the inability to identify these cases as high-risk were normal laboratory data and documentation problems in the BMP. ${ }^{9}$ Therefore, requiring EPs to complete more detailed patient history, physical examinations and laboratory findings as a basic requirement is likely to help prevent adverse events. Most importantly, EPs should make final decisions only when the evaluation process, including observation of symptoms and of treatment response, is complete. $^{12}$

Second, our study provided a database, patterns and causes of CSAEs in the ED, and a data collection process was applied in some reports. Data collected from such databases allow for the identification of patterns of medical errors and analyses of these data are important tools in studies on patient safety. ${ }^{16} 17$ Our CSAEs rate was $0.80 \%(165 / 20512$, $95 \%$ CI $0.75 \%$ to $0.95 \%)$, which was comparable with the rate of $0.71 \%$ in our previous report $(54 / 7646,95 \%$ CI $0.54 \%$ to $0.93 \%) .{ }^{9}$ Fordyce et al reported an error rate of $18 \%$, but the significant adverse event rate also included $0.36 \%$ of registered patients among the ED patients. ${ }^{11}$ In addition, Forster et al reported a $6 \%$ adverse event rate in the ED-discharged patients, but many of them were mild events. ${ }^{2}$ 
Table 4 Subjective reasons for discharging patients with CSAEs and objective review to define the causes of CSAEs in discharged patients ( $\mathrm{n}=165$ )

\begin{tabular}{llc}
\hline Emergency physicians' subjective reasons & Objective review to define the causes & Insufficiency of history record \\
\hline Mild diseases & 59 & Insufficiency of physical examinations \\
Improved symptoms & 53 & Insufficiency of laboratory/radiological examinations \\
Patients' wish to be discharged (not 'discharge against advice') & 22 & Observation of symptom \\
Normal laboratory and/or radiological examinations & 20 & Observation of treatment response \\
The patient can be followed up in the outpatient clinic & 7 & Judgement defect \\
Normal physical examinations & 4 & 32 \\
Consultant's opinions & 10 & 1 \\
\hline
\end{tabular}

CSAE, clinically significant adverse events.

Some cases had more than one cause.

Some specific findings and implications are summarised. First, in the study, evaluating the CSAEs through reviewing inadequacies in the BMP made the CSAEs more explainable, instead of just attributing them to judgement defect or the disease process. Liaw et al reported a rate of $79 \%$ of return-visit adverse events that were related to the disease process. ${ }^{18}$ Our results were different from the results of Liaw et al. That clear-cut evidence of inadequacies could be precisely defined and more easily found means auditing the BMP is an efficient way of assessing quality. In evaluating clinically adverse events/errors, judgement or disease process is always considered the cause if we cannot find a definite cause. When we check these adverse events/errors through the BMP, a systemic way, a definite cause is more easily found and a pure judgement or disease process becomes a less frequently occurring cause. This leaves fewer cases with poor outcomes considered unexplainable. Second, in our study we found that there was a gap between the subjective impression of EPs (who saw the patients with CSAEs) and the objective review of these CSAEs by senior EPs. Given that, a majority of cases with CSAEs were thought to have mild diseases, improved symptoms and a high certainty level of safety by the EPs who discharged them, this disparity may have instructive value. It is possible that the EPs were saying the patients were safe for discharge because they were rationalising it to themselves. It might be difficult to change EPs' judgment just before or when they discharge ED patients, so a better approach would be to make fundamental changes to the ways in which emergency medicine is practiced.

\section{Clinical implications}

Some improvements in processes can be developed. First, if the judgment of EPs as to the time when patients should be discharged is resistant to change, it would be more effective to modify the basic aspects of the evaluation process. Given that $98 \%$ of errors did not have a significant adverse event, ${ }^{11}$ the error rate of the ED's BMP in all ED patients, including those without adverse events, could be extremely high. Only after checking for the presence of clinical evidence and the completeness of the evaluation should EPs make their decision. A too direct and subjective decision process should be avoided. This is compatible with and could be enhanced by the notion of metacognition. ${ }^{15}$ Second, overcrowding is very common in many EDs and definitely affects EPs' ability to complete the BMP and that may worsen the quality of care in the ED during busy hours. A process involving the evaluation of ED patients and auditing adverse events in overcrowded conditions in the BMP cannot be overemphasised.

\section{Limitations}

First, EPs who saw return visit patients at their first visits might still have been able to recall the clinical conditions of the return visit patients during the interview or they may have learnt the information from their colleagues or from the computerised information system and then changed their responses in the interview, but without overall bias effect. Second, we asked EPs to give only one main reason for discharging the patients and this might have been problematic for EPs who wished to express multiple reasons. Third, failure to complete the interview was a potential problem due to the large number of interviews in our study, especially at the end of each month. However, there were only a few incomplete interviews. In order to overcome this problem, we did an intensive follow-up after each shift change, which was a more convenient time for interviews. Fourth, the intrinsic nature of the return visit could be problematic. However, the missed cases of return visits and visits to other hospitals were likely small in number. In another study, most cases with a CSAE returned to the ED within 3 days. ${ }^{9} 19$

\section{CONCLUSION}

The EPs who discharged patients subjectively thought that the majority of patients with CSAEs had mild diseases/clinical conditions However, a review of these cases found that the EPs had not done sufficient clinical follow-up or adequately performed basic clinical evaluations in most cases. It may therefore be difficult to change the subjective reasoning of EPs. Therefore, we recommend that EP follow-up of the BMPs (including history record, physical examination, laboratory and radiological examinations, clinical symptoms/signs and treatment) using clinical evidence as a guideline should be made mandatory.

Contributors L-MW and C-HC contributed to the study concept and design and take responsibility for the whole paper. C-HC and SS provided supervision and administrative support. C-HC provided funding. L-MW and C-KH were responsible for acquisition of data. M-CY designed, analysed and interpreted the data and provided statistical expertise. L-MW and C-HC drafted the manuscript. SS contributed to critical revision of the manuscript.

Funding This study was supported by a grant from the National Science Council, Taiwan, ROC (NSC: 94-2314-B-075-112).

\section{Competing interests None.}

Ethics approval This study was approved by the institutional review board of Taipei Veterans General Hospital, Taiwan.

Provenance and peer review Not commissioned; internally peer reviewed.

Open Access: This is an Open Access article distributed in accordance with the Creative Commons Attribution Non Commercial (CC BY-NC 3.0) license, which permits others to distribute, remix, adapt, build upon this work non-commercially, and license their derivative works on different terms, provided the original work is properly cited and the use is non-commercial. See: http://creativecommons.org/ licenses/by-nc/3.0/

\section{REFERENCES}

1. Stiell A, Forster AJ, Stiell IG, et al. Prevalence of information gaps in the emergency department and the effect on patient outcomes. CMAJ 2003;169:1023-8.

2. Foster AJ, Rose NG, van Walraven $C$, et al. Adverse events following emergency department visits. Qual Saf Health Care 2007;16:17-22.

3. Croskerry P. Achieving quality in clinical decision making: cognitive strategies and detection of bias. Acad Emerg Med 2002;9:1184-204. 
4. Brennan TA, Leape LL, Laird NM, et al. Incidence of adverse events and negligence in hospitalized patients. Results of the Harvard Medical Practice Study I. N Eng/ J Med 1991;324:370-6.

5. Thomas EJ, Studdert DM, Burstin HR, et al. Incidence and types of adverse events and negligent care in Utah and Colorado. Med Care 2000;38:261-71.

6. Rusnak RA, Borer J, Fastow JS. Misdiagnosis of acute appendicitis: common features discovered in cases after litigation. Am J Emerg Med 1994;12:397-402.

7. Espinosa JA, Nolan TW. Reducing errors made by emergency physicians in interpreting radiographs: longitudinal study. BMJ 2000;320:737-40.

8. Wolff AM, Bourke J. Detecting and reducing adverse events in an Australian ruralbased hospital emergency department using medical screening and review. Emerg Med J 2002;19:35-40.

9. Chern CH, How CK, Wang LM, et al. Decreasing clinically significant adverse events using feedback to emergency physicians of telephone follow-up outcomes. Ann Emerg Med 2005;45:15-23.

10. Elshove-Bolk J, Simons M, Cremers J, et al. A description of emergency department-related malpractice claims in the Netherlands: close claims study 1993-2001. Eur J Emerg Med 2004;11:247-50.

11. Fordyce J, Blank FSJ, Pekow P, et al. Errors in a busy emergency department. Ann Emerg Med 2003;42:324-33.
12. Kachalia AB, Gandhi TK, Puopolo AL, et al. Missed and delayed diagnosis in the emergency department: a study of closed malpractice claims from four liability insurers. Ann Emerg Med 2007:49:195-205.

13. Chern CC, Huang YT, Wang LM, et al. Are our emergency department practices really safe? Legal risks in the emergency department management process. Ann Emerg Med 2000;36:S65. Abstract.

14. Hockberger RS, Binder LS, Graber MA, et al. American College of Emergency Physicians Core Content Task Force II: the model of the clinical practice of emergency medicine. Ann Emerg Med 2001;37 745-70.

15. Croskerry P. Cognitive forcing strategies in clinical decision making. Ann Emerg Med 2003:41:110-20.

16. Shaw R, Drever $\mathrm{F}$, Hughes $\mathrm{H}$, et al. Adverse events and near miss reporting in the NHS. Qual Saf Health Care 2005:14:279-83.

17. Calder LA, Forster A, Nelson M, et al. Adverse events among patients registered in high-acuity areas of the emergency department: a prospective cohort study. CJEM 2010;12:421-30

18. Liaw SJ, Bullard MJ, Hu PM, et al. Rates and causes of emergency department revisits within 72 hours. J Formos Med Assoc 1999;98:422-5.

19. Hu SC. Analysis of patient revisits to the emergency department. Am J Emerg Med 1992;10:366-70. 